\title{
Nonthermal symmetry-broken states in the strongly interacting Hubbard model
}

\author{
Philipp Werner, ${ }^{1}$ Naoto Tsuji, ${ }^{1}$ and Martin Eckstein ${ }^{2}$ \\ ${ }^{1}$ Department of Physics, University of Fribourg, 1700 Fribourg, Switzerland \\ ${ }^{2}$ Max Planck Research Department for Structural Dynamics, University of Hamburg-CFEL, Hamburg, Germany
}

(Received 3 August 2012; published 1 November 2012)

\begin{abstract}
We study the time evolution of the antiferromagnetic order parameter after interaction quenches in the Hubbard model. Using the nonequilibrium dynamical mean-field formalism, we show that the system, after a quench from intermediate to strong interaction, is trapped in a nonthermal state which is reminiscent of a photodoped state and protected by the slow decay of doublons. If the effective doping of this state is low enough, it exhibits robust antiferromagnetic order, even if the system is highly excited and the thermal state is thus expected to be paramagnetic. We comment on the implication of our findings for the stability of nonthermal superconducting states.
\end{abstract}

DOI: 10.1103/PhysRevB.86.205101

PACS number(s): 71.10.Fd

\section{INTRODUCTION}

The rapid progress in the development of time-resolved spectroscopies enables direct measurements of dynamical symmetry breaking or the melting of long-range order in correlated materials. ${ }^{1}$ Ultrafast demagnetization after femtosecond optical pulses was first observed in ferromagnetic $\mathrm{Ni}^{2}$ The transient closing of the charge density wave (CDW) gap and excitations of CDW amplitude oscillations in $1 \mathrm{~T}-\mathrm{TaS}_{2}$ (Refs. 3-7) and $\mathrm{TbTe}_{3}$ (Ref. 8) were extensively studied using time-resolved photoemission. These experiments showed that the CDW gap disappears within less than 100 femtoseconds (essentially the time resolution of the experiment). Reference 9 reported the melting of orbital order and related structural transitions in manganites after a strong laser pulse. While the above studies focus on the destruction of some longrange-ordered state (or at least the associated gap in the electronic spectrum) by a strong excitation, and emphasize the fast timescale on which this process happens, there have also been recent experiments on photostimulated cuprates, which indicate the emergence of a nonthermal superconducting state which appears to be remarkably stable. ${ }^{10}$

Since all of these experiments involve transition-metal compounds, they raise fundamental questions about the dynamics of symmetry breaking transitions and the stability of symmetry-broken states in strongly interacting electron systems. While the concept of a phase transition is well understood in equilibrium, it is not obvious how to apply these ideas if a transition occurs under nonequilibrium conditions. For example, it is unclear whether and how the timescale for the melting of (quasi-) long-range order is related to equilibrium correlation times. A recent theoretical study of a dynamical Kosterlitz-Thouless transition found an evolution through nonthermal (superheated) states after a quench, and showed that the transition to a disordered state can become very slow if the latter is close to the equilibrium phase transition. ${ }^{11}$ This example indicates that a strongly excited nonequilibrium state can differ from a high-temperature disordered state on long timescales.

In this paper, we explore the dynamics of a symmetry-broken state in the most fundamental model for correlated electron materials, the Hubbard model, after an interaction quench in the strongly correlated regime.
More specifically, we consider the antiferromagnetically ordered state in the half-filled repulsive model, which (using a particle-hole transformation) can be mapped onto the $s$-wave superconducting state of the half-filled attractively interacting model. Our main finding is that a strong excitation of the system does not necessarily result in a rapid melting of the order parameter, even when the energy increase is sufficient to heat the system well above the Néel temperature in thermal equilibrium. Instead, the system can be trapped for a very long time in a nonthermal symmetry-broken state. We identify this metastable state as a state with an enhanced (nonthermal) number of doublons and holes, the lifetime of which depends exponentially on the interaction strength.

\section{MODEL AND METHOD}

The Hubbard Hamiltonian with time-dependent interaction is given by

$$
H(t)=\sum_{i j, \sigma} V_{i j} c_{i \sigma}^{\dagger} c_{j \sigma}+U(t) \sum_{i}\left(n_{i \uparrow}-\frac{1}{2}\right)\left(n_{i \downarrow}-\frac{1}{2}\right),
$$

where $V_{i j}$ is the hopping amplitude between sites $i$ and $j, \sigma$ is the spin index, and the local interaction between electrons of opposite spin is $U$. We choose the hoppings such that the density of states becomes semielliptical, $\rho(\epsilon)=$ $\sqrt{4 V^{2}-\epsilon^{2}} /\left(2 \pi V^{2}\right)$, and restrict our study to half-filling. Energy is measured in units of $V$ and time in units of $V^{-1}$. To solve this model, we employ the dynamical mean-field (DMFT) approximation, ${ }^{12}$ which has been extensively used to characterize the equilibrium phase diagram, and gives a qualitatively correct description for lattices with large coordination number. ${ }^{13}$ The DMFT formalism has recently been reformulated for nonequilibrium situations, ${ }^{14,15}$ which gave insights into quench dynamics in the Hubbard model, ${ }^{16,17}$ and to nonequilibrium states induced by external electric fields. ${ }^{18-23}$

Nonequilibrium DMFT studies up to now have been restricted to the paramagnetic phases of the model, although the extension of DMFT to symmetry-broken phases is straightforward. ${ }^{12}$ If the symmetry between spin-up and spindown Green's functions is not enforced, then the (single-site) DMFT phase diagram for the half-filled, repulsive Hubbard 

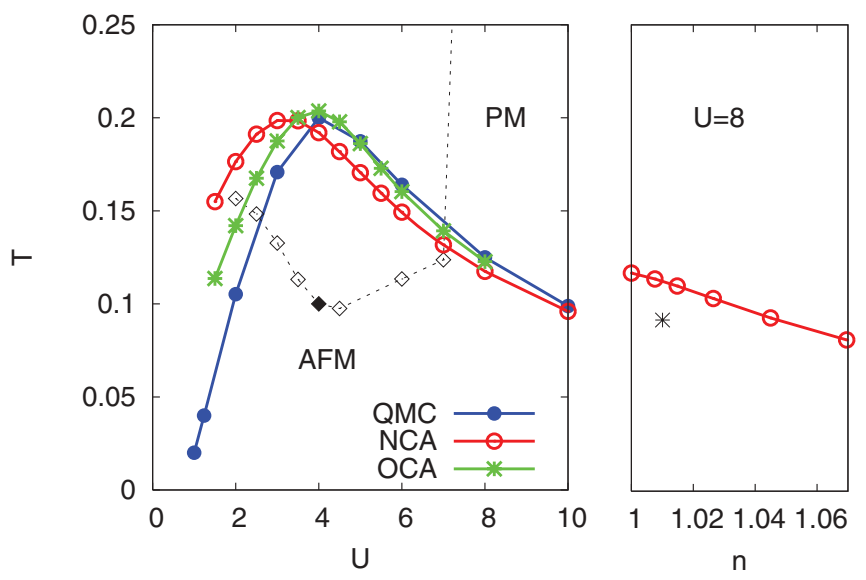

FIG. 1. (Color online) Left panel: Antiferromagnetic phase diagram for the half-filled Hubbard model. The blue line with circles shows the exact DMFT result (from Ref. 26), the red line with open circles the NCA approximation, and the green line with stars the OCA approximation. Diamonds indicate $T_{\text {eff }}(U)$ for interaction quenches from $U=4, T=0.1$. Right panel: NCA phase boundary as a function of filling at $U=8$. The star indicates the effective temperature of the doped Hubbard model with the same magnetization as in the trapped state (see text).

model exhibits an antiferromagnetically ordered phase at low temperature (denoted by AFM in Fig. 1). For attractive $U$, one finds an analogous phase diagram with AFM order replaced by $s$-wave superconductivity (at half-filling, the superconducting state is degenerate with a CDW phase, but in the doped system, superconductivity is more stable). The nature of the $s$-wave superconducting (or AFM insulating) state changes qualitatively as $|U|$ crosses the value corresponding roughly to the maximum in the critical temperature. This is known as the "BCS-BEC" crossover. ${ }^{24-26}$ Here, we will focus on the strongly interacting (non-Slater) AFM insulator, which corresponds to the "BEC" regime on the attractive side (for a complementary study of the weak-coupling or "BCS" regime, see Ref. 27). We are interested in the stability of the ordered phase after a sudden switch of $U$ into the paramagnetic regime. The quench provides an idealized but theoretically well-controlled excitation procedure. Although it is artificial from the point of view of condensed matter experiments, we will see in the following that the main effect of a quench to strong $U$ is to "freeze in" an excess number of doublons and holes (relative to the new equilibrium state), so that the dynamics is qualitatively similar to the behavior expected in photodoping experiments. ${ }^{28}$

To study antiferromagnetic states within DMFT on a bipartite lattice, we have to solve impurity problems for each sublattice. For the semielliptic density of states (Bethe lattice), the hybridization function $\Lambda_{A, \sigma}\left(\Lambda_{B, \sigma}\right)$ for the $A$ (B) sublattice is given by the self-consistency condition $\Lambda_{A, \sigma}=V^{2} G_{B, \sigma}\left(\Lambda_{B, \sigma}=V^{2} G_{A, \sigma}\right)$, where $G$ is the local lattice Green's function. Together with the relation $\Lambda_{A, \sigma}=$ $\Lambda_{B, \bar{\sigma}}$ (for pure Néel-type symmetry breaking), this leads to a single impurity calculation with self-consistency $\Lambda_{\sigma}=V^{2} G_{\bar{\sigma}}$. To solve the nonequilibrium DMFT impurity problem, we use self-consistent strong-coupling perturbation theory. Figure 1 shows the equilibrium phase diagram obtained with the two lowest-order implementations: the noncrossing approximation (NCA) (Ref. 29) and the one-crossing approximation (OCA) (Ref. 30). While NCA provides a rather good description of the exact quantum Monte Carlo (QMC) phase boundary ${ }^{26}$ in the strongly correlated regime, the maximum is shifted to $U_{\max }^{\mathrm{NCA}} \approx 3$ (while $U_{\max }^{\mathrm{QMC}} \approx 4$ ) and the phase boundary in the weakly correlated regime is not well reproduced. OCA brings a significant improvement, with an almost correct position of the maximum and a quantitatively accurate description of the phase boundary in the strong correlation regime. Since we are mainly interested in quenches within the strongly correlated regime, we will use the NCA method for the real-time calculations. The techniques for the solution of the nonequilibrium DMFT equations and our implementation of the real-time NCA/OCA impurity solver have been explained in detail in Refs. 17 and 31.

We measure the time-dependent expectation values of the magnetization $m=n_{\uparrow}-n_{\downarrow}$, the double occupancy $d=n_{\uparrow} n_{\downarrow}$ [which yields the local energy $E_{\text {loc }}=U d-\mu\left(n_{\uparrow}+n_{\downarrow}\right)$ ], and the kinetic energy per spin $E_{\text {kin, } \sigma}=-\frac{i}{L} \sum_{i j} V_{i j} G_{j i, \sigma}^{<}(t, t)$ $\left[G_{j i, \sigma}^{<}\left(t, t^{\prime}\right)=i\left\langle c_{i, \sigma}^{\dagger}\left(t^{\prime}\right) c_{j, \sigma}(t)\right\rangle\right]$. The latter can be expressed (within DMFT) as a convolution of the local Green's function $G_{i i, \sigma}$ and the hybridization function $\Lambda_{i, \sigma}$ : With $\Sigma_{i, \sigma}$ denoting the self-energy for site $i$, we can write the lattice and impurity Dyson equations as $\left[i \partial_{t}+\right.$ $\left.\mu-\Sigma_{i, \sigma}\right] G_{i j, \sigma}-\sum_{k} V_{i k} G_{k j, \sigma}=\delta_{i j}$ and $\left[i \partial_{t}+\mu-\Sigma_{i, \sigma}-\right.$ $\left.\Lambda_{i, \sigma}\right] G_{i i, \sigma}=1$. Hence, $\sum_{k} V_{i k} G_{k i, \sigma}=\Lambda_{i, \sigma} G_{i i, \sigma}$, and with sublattice indices $A$ and $B$ one obtains

$$
E_{\text {kin }, \sigma}=-(i / 2)\left[\Lambda_{A, \sigma} G_{A, \sigma}+\Lambda_{B, \sigma} G_{B, \sigma}\right]^{<}(t, t) .
$$

\section{RESULTS}

\section{A. Magnetization and spectral function}

The top panel of Fig. 2 plots the time evolution of the magnetization for quenches from an initial equilibrium state at $U=4$ and $T=0.1$, which is located deep inside the antiferromagnetic phase, to final states with $U=6,7,8$. The sudden increase of the interaction from $U(t=0)=U_{\text {initial }}$ to $U(t>0)=U_{\text {final }}$ causes a change in the total energy $E_{\mathrm{tot}}=E_{\mathrm{loc}}+\sum_{\sigma} E_{\mathrm{kin}, \sigma}$ of the system. $E_{\mathrm{tot}}$ stays constant after the quench (i.e., for $t>0$ ) and allows us to define an effective temperature $T_{\text {eff }}\left(U_{\text {final }}\right)$ corresponding to the temperature of the thermal state with interaction $U=U_{\text {final }}$ and total energy $E_{\mathrm{tot}}(t>0)$ :

$$
\frac{\operatorname{Tr}\left[H \exp \left(-H / T_{\text {eff }}\right)\right]}{\operatorname{Tr}\left[\exp \left(-H / T_{\text {eff }}\right)\right]}=E_{\text {tot }} \quad(\text { for } t>0) .
$$

This effective temperature is indicated by the black diamonds in Fig. 1. While $T_{\text {eff }}$ initially decreases with increasing $U$ due to the decreasing slope of constant entropy curves in this part of the phase diagram, ${ }^{32}$ it increases above $U \approx 4.5$ and crosses the AFM phase boundary slightly above $U=7$. After the quench to $U=8$, the system is expected to thermalize in a paramagnetic $(\mathrm{PM})$ state at high temperature $\left[T_{\text {eff }}(U=\right.$ $8)=0.732$ ]. However, as shown in Fig. 2, after a modest decrease the magnetization remains stuck for $t \gtrsim 20$ at some large, nonthermal value, with no further relaxation evident on the timescales accessible in our simulations. Similarly, the quenches to $U=6$ and 7 lead to a trapping in a nonthermal 

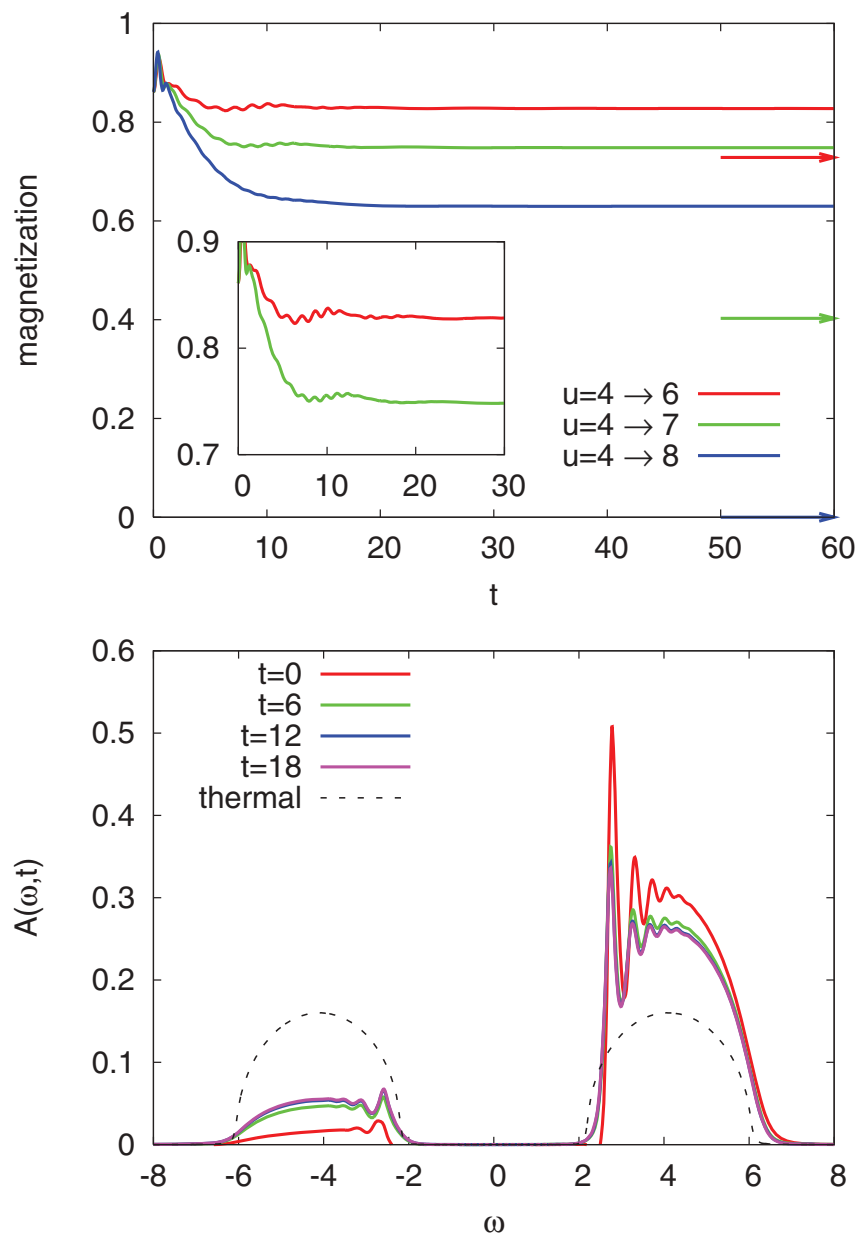

FIG. 2. (Color online) Top panel: Time evolution of the magnetization for quenches from $T=0.1$ and $U=4$ to $U=6,7$, and 8 . The effective temperatures after these quenches are $T_{\text {eff }}=0.113,0.124$ and $T=0.732$, respectively, and the arrows indicate the corresponding thermal values of the magnetization. Inset: Same data on a different scale. Bottom panel: Time evolution of $A(\omega, t)$ for the minority spin after a quench from $T=0.1$ and $U=4$ to $U=8$, and comparison to the thermal result (dashed). The pink curve $(t=18)$ corresponds to the spectral function of the long-lived nonthermal state.

state, as is evident from the comparison to the thermal value of the magnetization (arrows). While the transient dynamics also shows interesting behavior, in particular slow oscillations in the amplitude of the order parameter (with period $\approx 10$ ), with superimposed rapid $1 / U$ modulations (see inset of Fig. 2), we will focus in this paper on the trapping phenomenon and the nature of the long-lived nonthermal state.

To further characterize this state, we compute a timeresolved "spectral function" $A(\omega, t)$ from the Fourier transform of the retarded Green's function $G^{\mathrm{ret}}\left(t, t^{\prime}\right)=-i \Theta(t-$ $\left.t^{\prime}\right)\left\langle\left\{c(t), c^{\dagger}\left(t^{\prime}\right)\right\}\right\rangle$ :

$$
A(\omega, t)=-\frac{1}{\pi} \operatorname{Im} \int_{t}^{\infty} d t^{\prime} e^{i \omega\left(t^{\prime}-t\right)} G^{\mathrm{ret}}\left(t^{\prime}, t\right) .
$$

This function is plotted for the minority spin in Fig. 2, for the quench from $U=4, T=0.1$ to $U=8$. While the red curve $(t=0)$ should not be confused with the spectral function of the initial equilibrium state, it exhibits Hubbard bands with pronounced spin-polaron peaks, as is typical for the AFM insulator. ${ }^{33}$ Consistent with the top panel of Fig. 2, the spin imbalance shrinks from $t=0$ to $t \approx 18$ and then becomes time independent for larger $t$. The spectral function of the trapped nonthermal state still features spin-polaron peaks, in contrast to the thermal spectral function at $T_{\text {eff }}=0.732$ (dashed lines). We conclude that despite the strong excitation of the system and the large amount of energy injected by the quench from $U=4$ to $U=8$, the antiferromagnetic order does not melt rapildy; instead, the system is trapped in a long-lived state with large magnetization, and with the typical spectral features of a magnetically ordered state.

\section{B. Double occupancy}

To understand the nature of this trapped state and the reason for its robustness, we consider the time evolution of the average number of doubly occupied sites. The top left panel of Fig. 3 shows the result for the quench from $U=4, T=0.1$ to $U=8$. There is a complicated transient regime (with $1 / U$ oscillations) up to $t \approx 20$ in which the double occupancy decreases from about 0.044 to 0.021 . At longer times, the double occupancy is stuck at 0.021 , even though the expected thermal value is 0.0115 and thus almost a factor of 2 lower. This finding is consistent with theoretical and experimental studies of cold-atom systems, ${ }^{34}$ and with a previous investigation of pump-excited paramagnetic Mott insulators ${ }^{19}$ which show
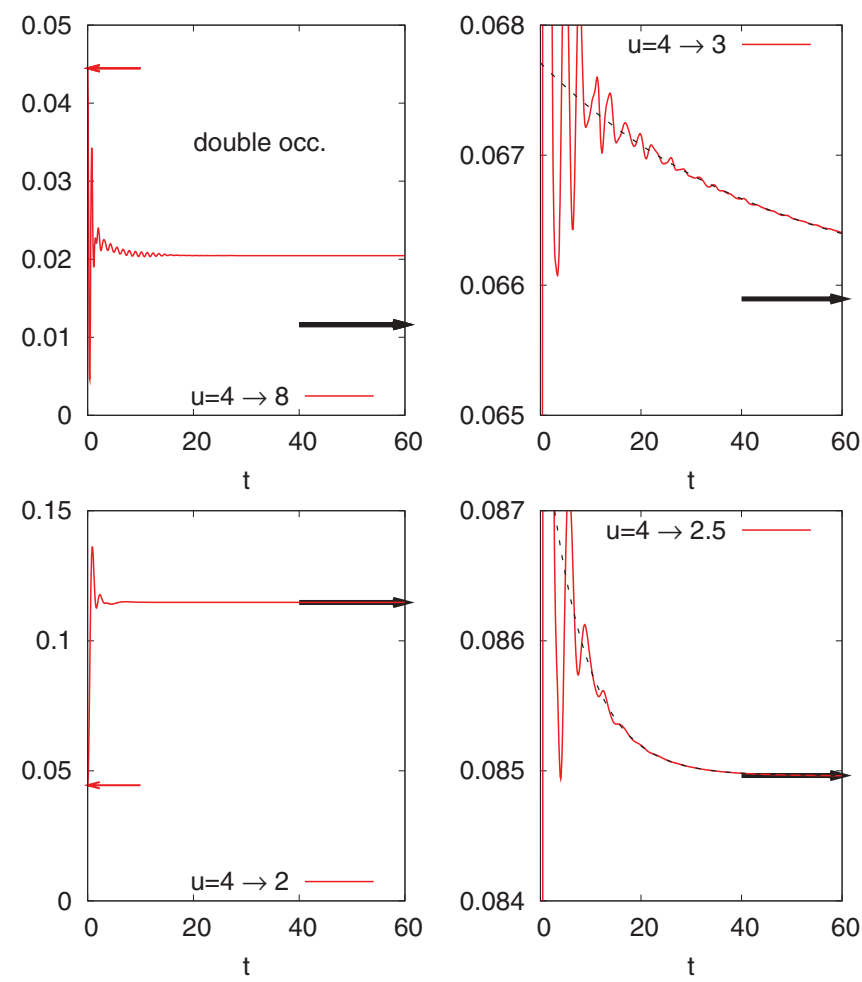

FIG. 3. (Color online) Time evolution of the double occupancy $\left\langle n_{\uparrow} n_{\downarrow}\right\rangle(t)$ for the quench from $U=4, T=0.1$ to $U=8,3,2.5,2$ (clockwise from the top left) and comparison to the thermal result (black arrow). The right panels show a narrow window around the thermal value and (as dashed line) an exponential fit to the long-time behavior. The left-pointing arrows in the left panels show the initial value of the double occupancy. 
that the relaxation time of doublons grows exponentially with $U$, leading (at large $U$ ) to a trapping in a nonthermal state characterized by a fixed number of doublons.

Indeed, if one quenches to smaller interaction, the relaxation time decreases and eventually becomes measurable on the timescales accessible in the simulations (right-hand panels of Fig. 3). We have fitted the long-time behavior of the double occupancy with an exponential function decaying onto the thermal value and thus extracted the relaxation times $\tau \approx 400$ for quenches from $T=0.1, U=4$ to $U=3.5, \tau \approx 47$ ( $U=$ $3)$, and $\tau \approx 7.8(U=2.5)$. The $U$ dependence of the relaxation time agrees with the analytical formula ${ }^{34} \tau=A e^{\alpha(U / 2) \ln (U / 2)}$, with $A=0.165$ and $\alpha=5.6$. For the paramagnetic phase studied in Ref. 19, a smaller coefficient $\alpha$ was found, but due to the tails in the Gaussian DOS used there, the results can not be directly compared by rescaling the hopping.

Around $U=2$, the relaxation becomes so fast that the system already thermalizes within the time of the initial transient (bottom left panel of Fig. 3). This finding of a qualitatively different relaxation pathway and fast thermalization at intermediate coupling is consistent with the results of Ref. 19 for pump excitations of the paramagnetic Mott insulator, and also with the result of Ref. 16 for interaction quenches from a noninteracting initial state.

\section{Comparison to a doped state}

The trapping of the double occupancy is well understood in the strong-coupling limit: For $U \gg V$, a unitary SchriefferWolff transformation $e^{S}$ can be constructed order-by-order in $V / U$, such that successively terms of all orders in $V / U$ are removed from the commutator $\left[e^{-S} H e^{S}, d\right]$, and hence $\bar{d}=e^{S} d e^{-S}$ is conserved on exponentially long times. ${ }^{35} \mathrm{At}$ second order, and after projection to $\bar{d}=0$, one would obtain the $t$ - $J$ model, but here we encounter a more general situation, with $\bar{d}>0$. One can consider $\bar{d}$ as a rigorous definition of the number of free doublons, which differs from $d$ by quantum fluctuations $[S, d]+\cdots=\mathcal{O}(V / U)$ (even in the Mott insulator at $T=0$, where $\bar{d}=0$ ). The initial very fast drop of $d(t)$ on the timescale of a few inverse hoppings (Fig. 3) is thus related to the reduction of quantum fluctuations due to the increase of $U$, or equivalently, a strengthening of the local moments. Because the quench is faster than the timescale of a quantum fluctuation, quantum fluctuations are transformed into real doublon and hole excitations with a certain amplitude. This gives rise to a nonzero $\bar{d}$ for $t>0$, and the stability of $\bar{d}$ on exponentially long times prevents the system from thermalization. The argument suggests that both the properties of the trapped magnetic states and the melting of the antiferromagnetic order can be related to the presence of injected free doublons and holes. To further support this fact, it is useful to take a closer look at the spectral function.

In Fig. 4, we compare the spectral function of the trapped state $(t=18)$ to equilibrium spectral functions of an infinitesimally doped $t-J$ model with $J=4 t^{2} / U$ and identical magnetization, as well as to a doped AFM Mott insulator. The spectral function for one hole in the $t-J$ model can be computed exactly within DMFT. ${ }^{36,37}$ The top panel of Fig. 4 reveals a good agreement of this $t$ - $J$ result and the spectral function of the half-filled Hubbard model at $U=8$, which
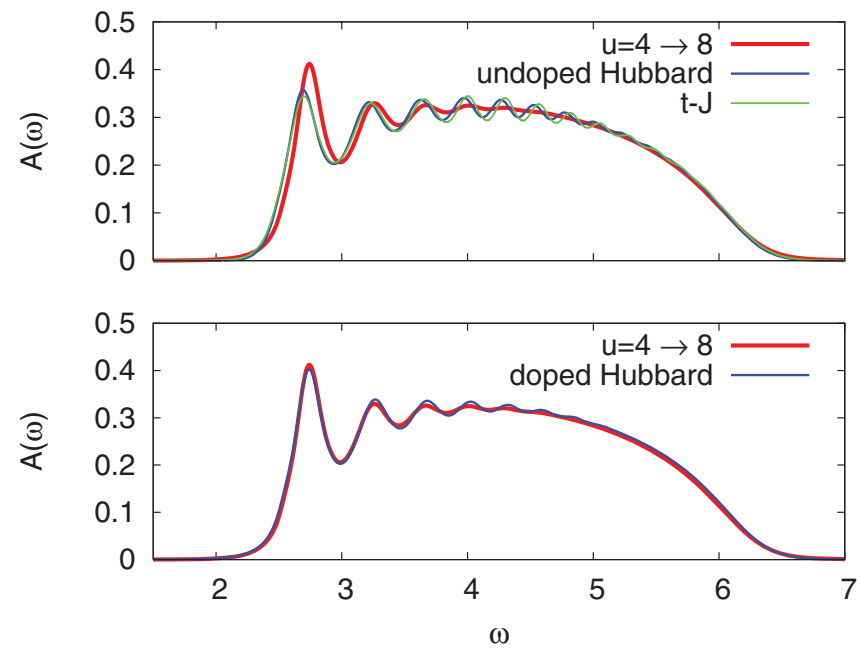

FIG. 4. (Color online) Comparison of the spectral function $A(\omega, t=18)$ of the trapped state after the quench from $U=4$, $T=0.1$ to $U=8$ (bold red line) to the minority-spin spectral function of a half-filled Hubbard model with $T=0.0986$ and to an infinitesimally doped $t$ - $J$ model with $J=4 t^{2} / U=0.5$ (top panel). The blue curve in the bottom panel corresponds to a doped Hubbard model with $T=0.0917$ and chemical potential chosen such that the magnetization and double occupancy of the trapped state are reproduced. (The $t-J$ and doped Hubbard spectra are shifted by +4.2 and +2.4 , respectively.)

shows that $U=8$ is in the $t$ - $J$ limit, and that the NCA solution of the Hubbard model in this interaction regime is reliable. (At nonzero temperature, the spectral functions of the half-filled and infinitesimally doped Hubbard model are identical.) The temperature $T=0.0986$ of the half-filled Hubbard model has been adjusted such that the magnetization corresponds to that of the trapped state, while the $t-J$ spectrum has been rescaled by $(1+m) / 2=0.815$, and the $t-J$ spectrum has been shifted on the frequency axis by +4.2 .

The figure also shows that the equilibrium results do not very well reproduce the peak structures in the spectral function of the trapped state. The first peak near the gap edge is more prominent, while the higher-energy peaks are smeared out. According to the above strong-coupling argument, the trapped state might be close to an equilibrium state with additional free doublons and holes. Such a state with doublons and holes is not accessible in thermal equilibrium within the Hubbard model. In the paramagnetic case and at low energies, its properties are different from a chemically doped state at the same doping. ${ }^{38}$ Nevertheless, because the interaction of doublons with other doublons is very different from the interaction of doublons with holes, one may assume that the main correction to the spectral function at positive energies (i.e., for inserting a doublon) is due to the presence of other free doublons, which motivates a comparison of the spectral function of the trapped state to an electron-doped equilibrium state. We determined the doping level $(n=1.010)$ and temperature $(T=0.0917)$ of the equilibrium Hubbard model such that the magnetization and double occupancy of the trapped state is reproduced. The corresponding point in the $T$-versus-filling phase diagram (right-hand panel of Fig. 1) is indicated by the black star symbol. The bottom panel of Fig. 4 shows that the spectral function 
of this doped Hubbard model (shifted on the frequency axis by +2.4 ) is much closer to that of the trapped state. In particular, the dominant first peak is well reproduced, while the damping of the peaks at higher energies is still not strong enough. This may be due to the missing interaction with holons.

\section{Occupation function}

Our results suggest that the trapped state is essentially an AFM Mott insulator with simultaneous electron and hole doping, comparable to a state that can be prepared by "photodoping." 39,40 The doping level is determined by the density of trapped doublons and holons and the effective temperature is determined by the trapped magnetization. In particular, the effective temperature of the trapped state after a quench from $U=4, T=0.1$ to $U=8$ is apparently below the Néel temperature $T_{N}^{\text {doped }}$ of this photodoped state. This remarkable fact must be the result of "entropy cooling": the reduction in the magnetization from 0.85 to 0.63 releases a lot of entropy and this in turn keeps the trapped state below its Néel temperature. Evidence for the cooling effect is provided by the occupied density of states

$$
A^{<}(\omega, t)=\frac{1}{\pi} \operatorname{Im} \int_{t}^{\infty} d t^{\prime} e^{i \omega\left(t^{\prime}-t\right)} G^{<}\left(t^{\prime}, t\right),
$$

which corresponds to a time-resolved photoemission spectrum for a quasi-steady state (see Fig. 5). Since doublons and holes are inserted locally, they initially have a large kinetic energy, which leads to a broad distribution of the weight of $A^{<}(\omega, t)$ in the upper Hubbard band. While the magnetization is decreasing, the weight becomes concentrated at low frequencies (near the lower band edge), indicating that doublons and holes are being cooled by exchanging energy with the spin background. This is in contrast to a quench in the paramagnetic phase, after which the occupation is not redistributed in time, ${ }^{19}$ because

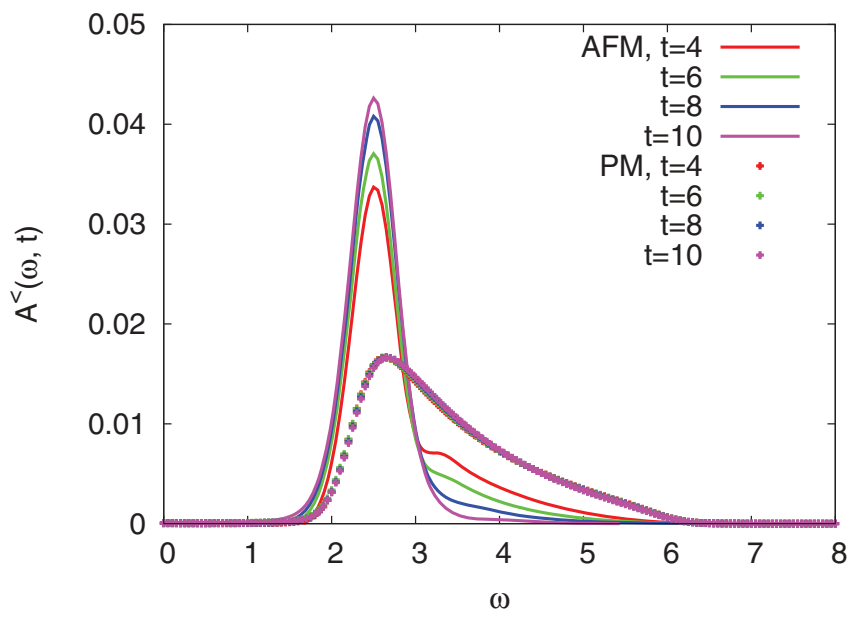

FIG. 5. (Color online) Time evolution of $A^{<}(\omega, t)$ for a quench from $U=4, T=0.1$ to $U=8$. While the occupied states rapidly accumulate at the lower band edge in the AFM calculation, the occupation is nearly time independent in the PM case. The curves are slightly broadened by introducing a Gaussian factor $e^{-\alpha\left(t-t^{\prime}\right)^{2}}$ in the integrand of Eq. (5), which ensures a smooth cutoff of the upper integration limit.
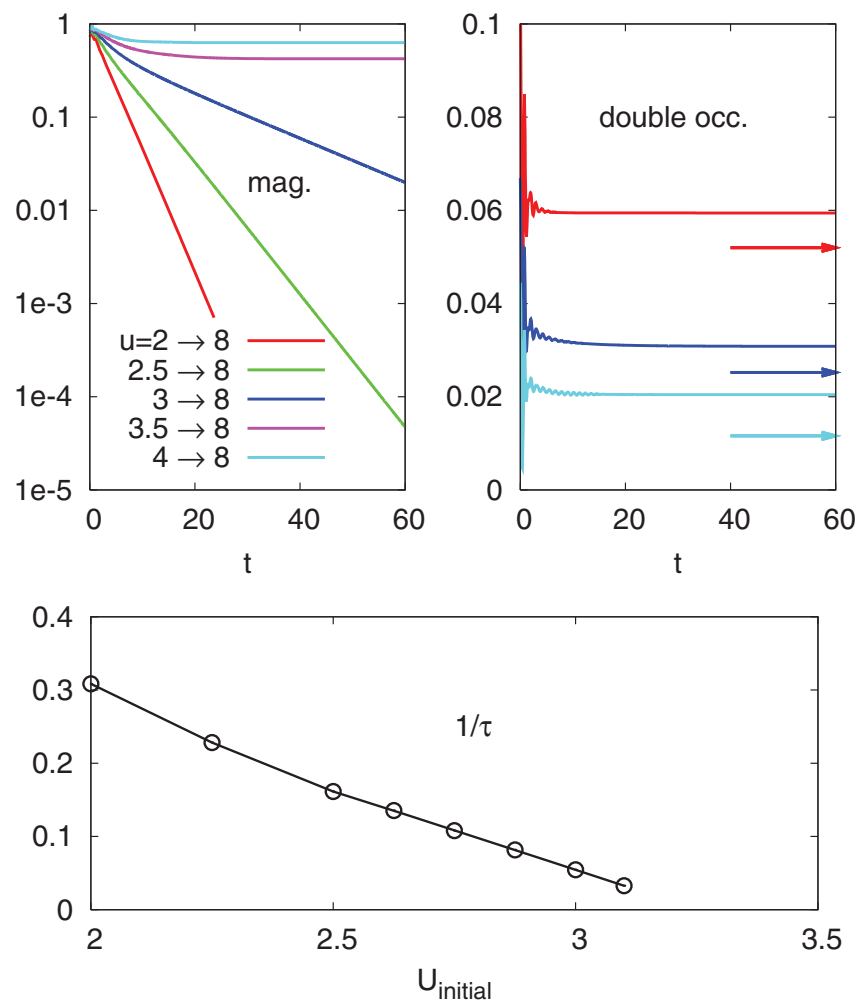

FIG. 6. (Color online) Time evolution of the magnetization (top left panel) and double occupancy (top right panel) for quenches from indicated initial $U, T=0.1$ to $U=8$. The bottom panel shows the inverse of the relaxation time $\tau$ as a function of initial $U$.

within DMFT the spin background of the paramagnetic state is completely disordered (no short-range correlations) and hence does not allow energy exchange with doublons and holes.

If the interpretation of entropy cooling is correct, one should be able to observe the transition of the trapped state from below $T_{N}^{\text {doped }}$ to above $T_{N}^{\text {doped }}$ in the nonequilibrium dynamics by changing the initial density of doublons and holes $\left(T_{N}^{\text {doped }}\right.$ decreases with doping, see right-hand panel of Fig. 1). Indeed, as illustrated in Fig. 6, the magnetization decays to zero exponentially for quenches from $U_{\text {initial }} \lesssim 3.25, T=0.1$ to $U=8$, while the system is trapped in magnetized states for quenches from $U_{\text {initial }} \gtrsim 3.25, T=0.1$. A smaller initial $U$ means a larger number of doublons and holes in the initial state, and thus also a larger doping of the effective photodoped state. In other words, around $U_{\text {initial }}=3.25$, the effective photodoped state crosses $T_{N}$. But, even for $U_{\text {initial }}<3.25$, where the AFM order decays to zero exponentially after the quench, the system is still trapped for very long times in a nonthermal state, as can be seen for example by comparing the double occupancy in the trapped state to the thermal value (top right panel of Fig. 6). The trapped state still corresponds to a photodoped state, but now at an effective temperature above $T_{N}^{\text {doped }}$. In this context, it is also interesting to look at the timescale $\tau$ for melting of the antiferromagnetic order, which diverges like $\tau^{-1} \propto U-U_{c}$ close to the transition (bottom panel of Fig. 6). This divergence resembles the critical slowing down at the equilibrium phase transition, which is related to the divergence of correlation times in equilibrium. 


\section{E. Implications for superconducting states}

Finally, let us comment on implications of these results for other symmetry-broken states, in particular, $s$-wave superconductivity (SC). As mentioned in the Introduction, the AFM phase of the half-filled repulsive Hubbard model can be mapped onto the $s$-wave SC phase in the half-filled attractive model. The mapping is a particle-hole transformation for the up spin on the bipartite lattice: $c_{i \uparrow} \rightarrow \tilde{c}_{i \uparrow}^{\dagger}$ for $i \in A, c_{i \uparrow} \rightarrow-\tilde{c}_{i \uparrow}^{\dagger}$ for $i \in B$, where $A$ and $B$ are sublattice indices. ${ }^{41}$ Applying this transformation to the repulsive model yields an attractive Hubbard model with $\tilde{U}=-U$. A photodoped antiferromagnet with doublons and holes transforms into a superconducting or charge-ordered state with an enhanced number of unpaired electrons. Our finding of long-lived nonthermal AFM states in the repulsive model therefore implies the existence of similar long-lived nonthermal SC states after quenches within the strongly interacting regime on the attractive side. The similarity of the trapped AFM state to a photodoped Mott insulator in turn suggests that a similar trapped SC state should appear after a photoinduced pair breakup in the strongly interacting attractive regime. The stability of this nonthermal SC is then linked to the slow recombination of unpaired electrons.

While it is clear that our simple model (with $s$-wave superconductivity) and the single-site DMFT formalism are in many respects not appropriate to describe the complicated physics of underdoped cuprates, one can speculate that some of the physics discussed in this paper may be at play in the recently reported experiment by the Cavalleri group, ${ }^{10}$ where a photoinduced transient SC state in a cuprate material was found to be stable for several tens of picoseconds, and thus much longer than the light-pulse which stimulated apical oxygens and (by a yet unknown mechanism) induced the SC state.

\section{CONCLUSIONS}

We have used the nonequilibrium DMFT formalism in combination with a NCA impurity solver to compute the time evolution of the antiferromagnetic order parameter in the strongly interacting Hubbard model, after a quench of the interaction parameter. Just as in the paramagnetic case, ${ }^{17}$ the system does not thermalize rapidly after the quench due to the long recombination time for doublons and holes. When the density of these excess doublons and holes is sufficiently low, the nonthermal transient state after the quench can exhibit antiferromagnetic order, with spectral features characteristic of a (doped) antiferromagnetic state, despite a strong heating effect, which will eventually lead to a thermalization of the system in a high-temperature paramagnetic state. The longlived, nonthermal antiferromagnetic state may be considered a manifestation of a "dark state," which does not exist in equilibrium, but can be induced by a suitable perturbation of the system. The observed behavior is very different from that of the antiferromagnetic Hubbard model at weak coupling, ${ }^{27}$ where doublons and holes are no longer stable, but qualitative features of the nonthermal Hartree solution are found to persist up to rather large values of the interaction.

While the interaction quench is convenient from a computational point of view, the main effect which we exploited in this study is the creation and freezing of excess doublons and holes in a strongly interacting antiferromagnetic insulator. Such charge carriers may be produced experimentally through photoexcitation. In connection with photodoping experiments, it is also relevant to note that the interaction with the antiferromagnetic background allows a rapid cooling of the excess doublons (within the doublon band), in contrast to the paramagnetic case where the distribution of excited carriers relaxes very slowly.

We have discussed the implications of our results for the possible observation of nonthermal superconducting states in strongly correlated systems. Long-lived, nonthermal symmetry broken states which are stabilized by the exponentially long lifetime of doublons should also affect the relaxation dynamics in other strongly correlated, long-range-ordered systems, such as CDW compounds. It might be particularly interesting to consider situations where different ordered phases compete or coexist and study the response to external perturbations. Finally, concerning the time evolution in antiferromagnetic insulators, it has recently been suggested that spin-polaron peaks are visible in the optical spectra of certain strongly correlated compounds, such as $\mathrm{LaSrMnO}_{4} \cdot{ }^{33}$ If this is indeed the case, then it might be possible to identify the nonthermal antiferromagnetic state (after photoexcitation) from signatures in a time-resolved photoemission spectrum.

\section{ACKNOWLEDGMENTS}

We thank H. Aoki, A. Georges, A. Lichtenstein, J. Mentink, A. J. Millis, and T. Oka for stimulating discussions. The calculations were run on the Brutus cluster at ETH Zurich. We acknowledge support from the Swiss National Science Foundation (Grant No. PP0022-118866) and FP7/ERC starting Grant No. 278023.

\footnotetext{
${ }^{1}$ A. L. Cavalieri, N. Müller, Th. Uphues, V. S. Yakovlev, A. Baltuka, B. Horvath, B. Schmidt, L. Blümel, R. Holzwarth, S. Hendel, M. Drescher, U. Kleineberg, P. M. Echenique, R. Kienberger, F. Krausz, and U. Heinzmann, Nature (London) 449, 1029 (2007). ${ }^{2}$ E. Beaurepaire, J.-C. Merle, A. Daunois, and J.-Y. Bigot, Phys. Rev. Lett. 76, 4250 (1996).

${ }^{3}$ L. Perfetti, P. A. Loukakos, M. Lisowski, U. Bovensiepen, H. Berger, S. Biermann, P. S. Cornaglia, A. Georges, and M. Wolf, Phys. Rev. Lett. 97, 067402 (2006).
}

${ }^{4}$ L. Perfetti, P. A. Loukakos, M. Lisowski, U. Bovensiepen, M. Wolf, H. Berger, S. Biermann, and A. Georges, New J. Phys. 10, 053019 (2008).

${ }^{5}$ J. C. Petersen, S. Kaiser, N. Dean, A. Simoncig, H. Y. Liu, A. L. Cavalieri, C. Cacho, I. C. E. Turcu, E. Springate, F. Frassetto, L. Poletto, S. S. Dhesi, H. Berger, and A. Cavalleri, Phys. Rev. Lett. 107, 177402 (2011).

${ }^{6}$ S. Hellmann, M. Beye, C. Sohrt, T. Rohwer, F. Sorgenfrei, H. Redlin, M. Kalläne, M. Marczynski-Bühlow, F. Hennies, 
M. Bauer, A. Föhlisch, L. Kipp, W. Wurth, and K. Rossnagel, Phys. Rev. Lett. 105, 187401 (2010).

${ }^{7}$ M. Eichberger, H. Schäfer, M. Krumova, M. Beyer, J. Demsar, H. Berger, G. Moriena, G. Sciaini, and R. J. D. Miller, Nature (London) 468, 799 (2010).

${ }^{8}$ F. Schmitt, P. S. Kirchmann, U. Bovensiepen, R. G. Moore, L. Rettig, M. Krenz, J.-H. Chu, N. Ru, L. Perfetti, D. H. Lu, M. Wolf, I. R. Fisher, and Z.-X. Shen, Science 321, 1649 (2008).

${ }^{9}$ P. Beaud, S. L. Johnson, E. Vorobeva, U. Staub, R. A. De Souza, C. J. Milne, Q. X. Jia, and G. Ingold, Phys. Rev. Lett. 103, 155702 (2009).

${ }^{10}$ D. Fausti, R. I. Tobey, N. Dean, S. Kaiser, A. Dienst, M. C. Hoffmann, S. Pyon, T. Takayama, H. Takagi, and A. Cavalleri, Science 331, 189 (2011).

${ }^{11}$ L. Mathey and A. Polkovnikov, Phys. Rev. A 81, 033605 (2010).

${ }^{12}$ A. Georges, G. Kotliar, W. Krauth, and M. J. Rozenberg, Rev. Mod. Phys. 68, 13 (1996).

${ }^{13}$ M. Metzner and D. Vollhard, Phys. Rev. Lett. 62, 324 (1989).

${ }^{14} \mathrm{P}$. Schmidt and H. Monien, arXiv:cond-mat/0202046.

${ }^{15}$ J. K. Freericks, V. M. Turkowski, and V. Zlatić, Phys. Rev. Lett. 97, 266408 (2006).

${ }^{16}$ M. Eckstein, M. Kollar, and P. Werner, Phys. Rev. Lett. 103, 056403 (2009).

${ }^{17}$ M. Eckstein, M. Kollar, and P. Werner, Phys. Rev. B 81, 115131 (2010).

${ }^{18}$ M. Eckstein, T. Oka, and P. Werner, Phys. Rev. Lett. 105, 146404 (2010)

${ }^{19}$ M. Eckstein and P. Werner, Phys. Rev. B 84, 035122 (2011).

${ }^{20}$ M. Eckstein and P. Werner, Phys. Rev. Lett. 107, 186406 (2011).

${ }^{21}$ N. Tsuji, T. Oka, P. Werner, and H. Aoki, Phys. Rev. Lett. 106, 236401 (2011).

${ }^{22}$ N. Tsuji, T. Oka, H. Aoki, and P. Werner, Phys. Rev. B 85, 155124 (2012).
${ }^{23}$ C. Aron, G. Kotliar, and C. Weber, Phys. Rev. Lett. 108, 086401 (2012).

${ }^{24}$ R. Micnas, J. Ranninger, and S. Robaszkiewicz, Rev. Mod. Phys. 62, 113 (1990).

${ }^{25}$ A. Toschi, M. Capone, and C. Castellani, Phys. Rev. B 72, 235118 (2005).

${ }^{26}$ A. Koga and P. Werner, Phys. Rev. A 84, 023638 (2011).

${ }^{27}$ N. Tsuji, M. Eckstein, and P. Werner, arXiv:1210.0133.

${ }^{28} \mathrm{We}$ have confirmed that the main conclusions of this paper can be reproduced with a photodoping setup, in particular, the trapping in a long-lived nonthermal antiferromagnetic state for small doping, and the fast relaxation to a (nonthermal) paramagnetic state in the case of large doping.

${ }^{29}$ H. Keiter and J. C. Kimball, Int. J. Magn. 1, 233 (1971).

${ }^{30}$ T. Pruschke and N. Grewe, Z. Phys. B 74, 439 (1989).

${ }^{31}$ M. Eckstein and P. Werner, Phys. Rev. B 82, 115115 (2010).

${ }^{32}$ A. Sotnikov, D. Cocks, and W. Hofstetter, Phys. Rev. Lett. 109, 065301 (2012).

${ }^{33}$ C. Taranto, G. Sangiovanni, K. Held, M. Capone, A. Georges, and A. Toschi, Phys. Rev. B 85, 085124 (2012).

${ }^{34}$ R. Sensarma, D. Pekker, E. Altman, E. Demler, N. Strohmaier, D. Greif, R. Jördens, L. Tarruell, H. Moritz, and T. Esslinger, Phys. Rev. B 82, 224302 (2010).

${ }^{35}$ A. H. MacDonald, S. M. Girvin, and D. Yoshioka, Phys. Rev. B 37, 9753 (1988).

${ }^{36}$ R. Strack and D. Vollhardt, Phys. Rev. B 46, 13852 (1992).

${ }^{37}$ D. E. Logan and M. P. H. Strumpf, Europhys. Lett. 43, 207 (1998).

${ }^{38}$ M. Eckstein and P. Werner, arXiv:1207.0402.

${ }^{39}$ S. Iwai, M. Ono, A. Maeda, H. Matsuzaki, H. Kishida, H. Okamoto, and Y. Tokura, Phys. Rev. Lett. 91, 057401 (2003).

${ }^{40}$ H. Okamoto, T. Miyagoe, K. Kobayashi, H. Uemura, H. Nishioka, H. Matsuzaki, A. Sawa, and Y. Tokura, Phys. Rev. B 82, 060513 (2010).

${ }^{41}$ H. Shiba, Prog. Theor. Phys. 48, 2171 (1972). 